\title{
Número de ramos plagiotrópicos e produtividade de duas cultivares de cafeeiro utilizando irrigação por gotejamento ${ }^{1}$
}

\author{
Number of primary branches and productivity of two cultivars of coffee using drip \\ irrigation
}

\author{
André Ribeiro da Costa ${ }^{2 *}$, Roberto Rezende ${ }^{3}$, Paulo Sérgio Lourenço de Freitas ${ }^{3}$, José Antônio Frizzone ${ }^{4}$ e Celso \\ Helbel Júnior ${ }^{5}$
}

\begin{abstract}
Resumo - Este experimento foi conduzido em uma área do Centro Técnico de Irrigação (CTI) da Universidade Estadual de Maringá (UEM) com o objetivo de avaliar o efeito da aplicação de porcentagens de doses para NPK, no número de ramos plagiotrópicos e na produtividade das cultivares de cafeeiro Obatã e IAPAR - 59 em diferentes cultivos (não irrigado, irrigado e fertirrigado). As mudas foram plantadas em espaçamento de 2,0 metros entre linhas e 1,0 metro entre plantas, caracterizando sistema adensado. Utilizou-se o sistema de irrigação localizada por gotejamento. O experimento foi esquematizado em delineamento inteiramente casualizado em parcelas subdivididas com quatro repetições. As parcelas e subparcelas foram compostas pelas porcentagens de doses de NPK e pelos cultivos, respectivamente. A interação entre estes fatores influenciou significativamente a produtividade e o número de ramos plagiotrópicos, permitindo concluir qual a dose de fertilizante mais indicada para cada cultivo. A produtividade máxima para a cultivar Obatã foi conseguida com as doses de NPK de percentual $119,5 \%, 151,25 \%$, e $154,17 \%$ para os cultivos não irrigados, irrigados e fertirrigados, respectivamente. A máxima produtividade da cultivar IAPAR - 59 nos cultivos não irrigados, irrigados e fertirrigados foi alcançada com os percentuais de 137,83\%, 162\% e 151,75\%, respectivamente. Em relação ao número de ramos plagiotrópicos, a melhor dose para a cultivar Obatã, nos cultivos irrigados e fertirrigados, correspondeu a dose de $200 \%$, enquanto que a dose de $150 \%$ foi a mais adequada para as plantas irrigadas da cultivar IAPAR - 59. Os melhores desempenhos produtivos foram obtidos nos cultivos fertirrigados.
\end{abstract}

Palavras-chave - Café. Água de irrigação. Irrigação por Gotejamento.

Abstract - This experiment was conducted in an area of the Technical Center of Irrigation (CTI) of the State University of Maringá (UEM) with the objective of evaluating the effect of the application of different percentages of doses of NPK (50\%; $100 \% ; 150 \% ; 200 \%$ ) in the number of plagiotropic branches and in the productivity of the cultivars of coffee Obatã and IAPAR - 59. The seedlings were planted in the spacing of 2.0 meters between lines and 1.0 meter within lines, characterizing a dense system. In this experiment was used the system of localized irrigation by dripping. The experiment followed a design completely randomized in split plots with four replications. The plots and subplots were composed by percentages of doses of NPK and crops, respectively. The interaction between those factors influenced significantly the productivity and the number of plagiotropic branches allowing concluding which dose of fertilizer is indicated for each cultivation. The maximum yield to grow Obatã was achieved with doses of NPK percentages of $119.5 \%, 151.25 \%$ and $154.17 \%$ for non-irrigated crops, irrigated and fertilized, respectively. The maximum yield for IAPAR - 59 in nonirrigated crops, irrigated and fertilized was achieved with doses of NPK percentages of $137.83 \%, 162 \%$ and $151.75 \%$ respectively. Regarding the number of primary branches the best dose for cultivating crops in irrigated and Obatã fertirrigated corresponded to a percentage of $200 \%$, while the percentage dose of $150 \%$ showed the most suitable for IAPAR - 59 for the irrigated crops. The best productive was obtained for the fertirrigated cultivations.

Key words - Coffee. Irrigation water. Drip irrigation.

\footnotetext{
* Autor para correspondência

${ }^{1}$ Recebido para publicação em 04/11/2009; aprovado em 04/10/2010

Pesquisa financiada pelo Conselho Nacional de Desenvolvimento Científico e Tecnológico - CNPq, extraída da dissertação de mestrado do primeiro autor

${ }^{2}$ Programa de Pós-Graduação em Agronomia, CCA/UEM, Rua Pioneiro Antônio de Paula Freitas, 154, Jardim Império do Sol, Maringá-PR, Brasil,

87.083-550, rcosta4@hotmail.com

${ }_{3}^{3}$ Departamento de Agronomia, CCA/UEM, Maringá-PR, Brasil, rrezende@uem.br, pslfreitas@uem.br

${ }^{4}$ Departamento de Engenharia Rural, ESALQ/USP, Caixa Postal 9, Piracicaba-SP, Brasil, 13.418-900, frizzone@esalq.usp.br

${ }^{5}$ Instituto Agronômico do Paraná, Londrina-PR, Brasil, jrhelbel@ibest.com.br
} 


\section{Introdução}

A utilização de irrigação nas lavouras cafeeiras favorece o pleno desenvolvimento e produção das plantas. A cafeicultura irrigada é uma atividade agrícola de muito destaque, em virtude de sua rentabilidade (COELHO et al.,2002). Caramori et al. (2001) apontam que o Estado do Paraná é apto a cafeicultura sem irrigação. Entretanto, segundo Mantovani et al. (2004), a implantação de projetos de irrigação, em áreas tradicionais de cafeicultura de sequeiro, trazem uma maior produtividade às lavouras, assim como melhor qualidade ao produto final.

Segundo Carvalho et al. (2006), a irrigação potencializa a produção de gemas e isto pode influenciar indiretamente a produção. Nazareno et al. (2003), em um experimento, avaliaram o número de ramos plagiotrópicos de cafeeiros conduzidos com e sem irrigação. A adoção da irrigação promoveu aumento de $16 \%$ no número de ramos plagiotrópicos. Bonomo et al. (2008) avaliaram a produtividade de cafeeiros conduzidos através de irrigação. Os resultados mostraram que a utilização das técnica de irrigação (aspersão ou gotejamento) proporcionou ganhos em nível de produtividade com maior destaque a cultivar Katipó.

A introdução de sistemas de irrigação localizada por gotejamento, na cafeicultura brasileira, ocorreu no início da década de 90 . Na segunda metade desta década, os sistemas de gotejamento se popularizaram e sua aplicação em lavouras de café cresceu de forma significativa (SILVA et al., 2003).

Segundo Santana et al. (2004), em sistemas de irrigação localizada, ocorre economia de água, uma vez que a água é aplicada com eficiência somente na região próxima a planta. Além disso, no primeiro ano de cultivo do cafeeiro, a evaporação da água do solo prevalece sobre a transpiração da cultura.

Santinato et al. (2008) citam as vantagens da utilização da irrigação localizada por gotejamento, como: a) maior eficiência no uso da água, na adubação e no controle fitossanitário; b) maior produtividade; c) menor interferência nas práticas culturais; d) boa adaptação a distintas topografias e solos; e) possibilidade de sua utilização em solos salinos e com água salina; f) economia de mão-de-obra.

A fertirrigação pode ser definida como uma combinação das práticas de fertilização e irrigação, ou seja, os adubos minerais são injetados na água de irrigação (VITTI et al., 1994), graças aos modernos sistemas de irrigação localizada que dispõem de equipamentos para a aplicação de fertilizantes químicos através da água de irrigação (ANDRADE et al., 2004).

Silva et al. (2005) afirmam que a fertirrigação apresenta algumas vantagens, tais como: a não compactação do solo e o fim de injúrias mecânicas as plantas, pois os maquinários pesados não ingressam mais na área de plantio para a realização da adubação convencional; menor quantidade de equipamento exigido; menor gasto de energia. Além disso, a dosagem dos fertilizantes é mais bem regulada, monitorada, parcelada e distribuída ao longo do perfil do solo de acordo com as necessidades da cultura no decorrer do seu ciclo fenológico.

Duenhas et al. (2002) afirmam que, apesar de existirem publicações sobre a aplicação de fertilizantes por meio da água de irrigação, existe a necessidade de pesquisas sobre doses, concentrações e parcelamentos dos nutrientes para as culturas, visto que esta técnica sofre a influência de diversas variáveis.

Este trabalho teve como objetivo avaliar a influência da aplicação de diferentes porcentagens de doses de NPK e de diferentes cultivos no número de ramos plagiotrópicos e na produtividade de duas cultivares de cafeeiro cultivadas no municipio de Maringá, Paraná.

\section{Material e métodos}

O presente trabalho foi realizado em uma área experimental do Centro Técnico de Irrigação (CTI) da Universidade Estadual de Maringá, localizado na cidade de Maringá - PR, cujas coordenadas geográficas são de $23^{\circ} 25^{\prime}$ de Latitude Sul e $51^{\circ} 57^{\prime}$ de Longitude Oeste. O relevo apresenta diferença de nível média de $6 \%$, podendo ser considerado como homogêneo e suavemente ondulado.

O clima da área é do tipo Cfa Mesotérmico Úmido, caracterizado por chuvas abundantes no verão e invernos secos, segundo Koppen. As médias de precipitação anual chegam a $1500 \mathrm{~mm}$. As médias das temperaturas mínimas e das máximas atingem $10,3{ }^{\circ} \mathrm{C}$ e $33,6^{\circ} \mathrm{C}$, respectivamente. A temperatura média anual chega a $16,7{ }^{\circ} \mathrm{C}$ e a umidade relativa média do ar é igual a $66 \%$.

$\mathrm{Na}$ Figura 1, estão dispostas as temperaturas máximas, mínimas e médias, registradas no decorrer do experimento. Santinato et al. (2008) citam que a faixa de temperatura média anual ótima para regiões aptas ao cultivo do café arábica situa-se entre 19 e $23^{\circ} \mathrm{C}$, sendo que os cafeeiros jovens toleram temperaturas médias maiores que este limite superior. 


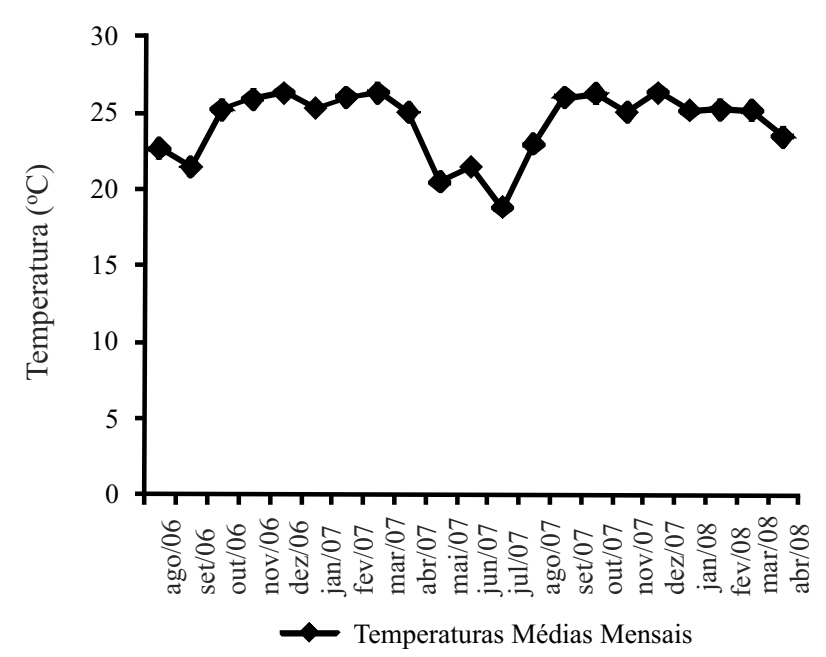

Figura 1 - Médias mensais de temperaturas máximas, médias e mínimas registradas no período compreendido entre agosto de 2006 e abril de 2008

Na Figura 2, são mostrados os totais mensais de precipitação e as lâminas mensais aplicadas de irrigação no decorrer do experimento. Nota-se que os maiores volumes de precipitação ocorreram no mês de setembro de 2006. No mês de agosto de 2007, não houve incidência de precipitações na área experimental.

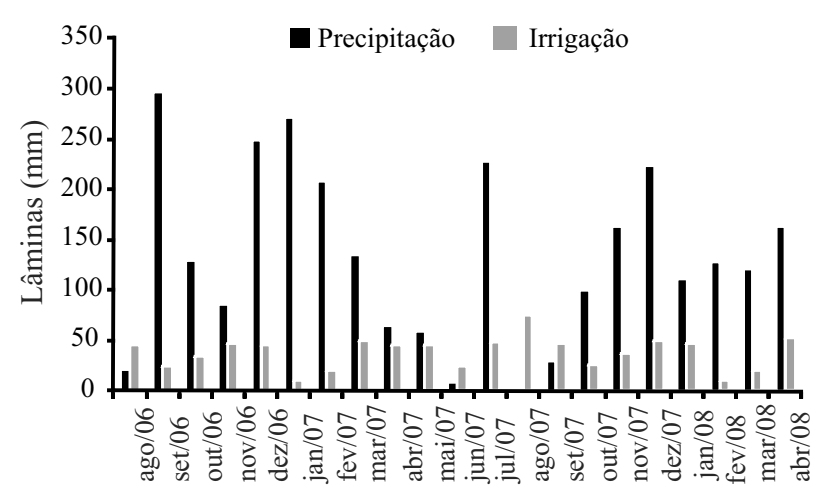

Figura 2 - Totais mensais de precipitação e lâminas mensais aplicadas de irrigação no período compreendido entre agosto de 2006 e abril de 2008

Realizou-se o plantio em dezembro de 2005. As mudas de cafeeiro pertencentes às cultivares IAPAR - 59 e em Obatã foram plantadas com espaçamento de 2,0 m entre linhas e 1,0 m entre plantas. Tal arranjo caracterizou um sistema adensado.
O solo da área pertence à classe Nitossolo Vermelho distroférrico com Horizonte A moderado, textura argilosa, fase florestal subperenifólia (EMBRAPA, 1999). O solo da área é de textura muito argilosa com $18 \%$ de areia, $7,5 \%$ de silte e $74,5 \%$ de argila e apresenta as seguintes características químicas: $\mathrm{pH}$ em $\mathrm{CaCl}_{2}, 5,2$; matéria orgânica, 2,17 dag $\mathrm{kg}^{-1}$; $\mathrm{P}$ disponível (Mehlich - 1), 1,4 $\mathrm{mg} \mathrm{dm}^{-3}$; teores de $\mathrm{K}^{+}, \mathrm{Mg}^{2+}$ e $\mathrm{Al}^{3+}$ de 0,$60 ; 2,57$ e $0,00 \mathrm{cmol}_{\mathrm{c}} \mathrm{dm}^{-3}$, respectivamente; acidez de troca $\left(\mathrm{H}^{+}+\mathrm{Al}^{3+}\right), 4,37 \mathrm{cmol}_{\mathrm{c}} \mathrm{dm}^{-3}$; CTC a pH 7,0 de 14,49 $\mathrm{cmol}_{\mathrm{c}} \mathrm{dm}^{-3}$ e saturação de bases (V\%) igual a $68,75 \%$.

Tendo-se a lavoura recém-formada e visando garantir o pegamento uniforme das mudas, procedeuse a irrigação através do sistema de irrigação localizada por gotejamento até a diferenciação dos tratamentos, que se iniciou em agosto de 2006. Para as operações de tratos culturais e controle fitossanitário, seguiu-se a recomendação de Matiello et al.(2005).

Para a adubação antes da diferenciação dos tratamentos, também se seguiu a recomendação de Matiello et al. (2005). Para o suprimento de fósforo, aplicou-se $250 \mathrm{~kg} \mathrm{ha}^{-1}$ de $\mathrm{P}_{2} \mathrm{O}_{5}$. Com relação ao potássio, o mesmo foi fornecido juntamente com a adubação nitrogenada, através da aplicação do formulado comercial 20-00-20, em doses de 10 gramas por planta em cada aplicação realizada.

Os emissores autocompensantes da marca Goldentrip, instalados na linha, operaram com vazão nominal e pressão de serviço de $1,2 \mathrm{~L} \mathrm{~h}^{-1}$ e 10 m.c.a, respectivamente. Os gotejadores foram instalados a uma distância de 0,2 $\mathrm{m}$ do caule das plantas, sobre a superfície do solo para que a superfície molhada pudesse formar uma faixa contínua ao longo da linha de plantio. Os gotejadores distaram 0,40 metros nas linhas de plantio e 2,0 metros entre elas.

Para o manejo da irrigação, recorreu-se a utilização do programa computacional IRRIPLUS, de modo que os dados climatológicos necessários para a alimentação deste software, tais como temperatura média, máxima e mínima, umidade relativa média do ar e precipitação foram obtidos diariamente junto a Estação Climatológica da Universidade Estadual de Maringá. Utilizou-se uma lâmina média de $6 \mathrm{~mm}$ ao longo do experimento.

A recomendação das doses de $\mathrm{N}$, de $\mathrm{P}$ e de $\mathrm{K}$ foi baseada em Matiello et al. (2005), que indicam a dose de $150 \mathrm{~kg} \mathrm{ha}^{-1}$ para $\mathrm{N}$ e $\mathrm{K}_{2} \mathrm{O}$, a qual corresponde à porcentagem de $100 \%$. Além desta dose, foram testadas doses inferiores $\left(75 \mathrm{~kg} \mathrm{ha}^{-1}\right)$ e doses superiores $\left(225\right.$ e $\left.300 \mathrm{~kg} \mathrm{ha}^{-1}\right)$, que equivalem aos percentuais de $50 \%, 150 \%$ e $200 \%$, respectivamente. Para o $\mathrm{P}$, a dose correspondente ao percentual de $100 \%$ é de $30 \mathrm{~kg} \mathrm{ha}^{-1}$ 
de $\mathrm{P}_{2} \mathrm{O}_{5}$. Deste modo, também foram avaliadas doses inferiores $\left(15 \mathrm{~kg} \mathrm{ha}^{-1}\right)$ e superiores (45 e $\left.60 \mathrm{~kg} \mathrm{ha}^{-1}\right)$, que correspondem às variações percentuais de $50 \%$, $150 \%$ e $200 \%$, respectivamente.

Os tratamentos aplicados às plantas de cada cultivar, utilizada neste trabalho, foram formados através da combinação das quatro doses para nitrogênio, fósforo e potássio, as quais foram anteriormente descritas com três cultivos (não irrigado, irrigado e fertirrigado), tendo-se um total de 12 tratamentos para cada cultivar, com cada um tendo quatro repetições, com dez plantas representando uma repetição.

Foi montado um experimento independente para cada cultivar. Utilizou-se o delineamento inteiramente casualizado, com parcelas subdivididas. As doses de NPK formaram as parcelas, por meio de sorteio, de modo que cada uma formou um setor da área do experimento. Posteriormente, no interior de cada setor, as subparcelas foram compostas pelas linhas de plantas, as quais receberam, através de sorteio, os três cultivos (não irrigado, irrigado e fertirrigado). As doses de NPK e os cultivos, bem como a interação entre estas fontes de variação, foram estudadas para cada cultivar num total de quatro repetições.

Os tratamentos ocorreram em linhas de plantas de 40 metros de comprimento, sendo que as parcelas foram compostas a partir destas perfazendo, cada quarenta plantas, uma parcela. As plantas das cultivares de cafeeiro IAPAR - 59 e Obatã foram dispostas em linhas intercaladas, possibilitando a condução do experimento dentro de uma mesma área, a qual foi de 0,8 hectares.

Cada subparcela foi constituída de um grupo de dez plantas, selecionadas aleatoriamente, nas linhas de plantio, tendo como bordadura as linhas de plantas periféricas da área experimental e as três plantas iniciais e finais de cada linha. Cada subparcela tinha uma linha de plantio.

Foram usadas como fontes de nitrogênio, de fósforo e de potássio, nos cultivos fertirrigados, nitrato de cálcio $(15 \%$ de $\mathrm{N})$, fosfato monomamônico $(50 \%$ de $\left.\mathrm{P}_{2} \mathrm{O}_{5}\right)$ e nitrato de potássio $\left(44 \%\right.$ de $\left.\mathrm{K}_{2} \mathrm{O}\right)$. Na adubação convencional, empregada nos cultivos irrigados e não irrigados, procedeu-se ao uso do formulado comercial 20-05-20 (20\% de N, 5\% de $\mathrm{P}_{2} \mathrm{O}_{5}$ e $20 \%$ de $\mathrm{K}_{2} \mathrm{O}$ ).

A única variação nos fertilizantes utilizados com a diferenciação dos tratamentos foi em relação à NPK, sendo os demais elementos aplicados da mesma maneira para todos os tratamentos.

Nas parcelas não irrigadas e irrigadas, a aplicação de NPK foi realizada convencionalmente em cobertura, parcelada em duas aplicações, durante o período chuvoso, em faixa, com distribuição manual ao redor das plantas.

As plantas conduzidas nos cultivos fertirrigados receberam as doses de $\mathrm{N}$, de $\mathrm{P}$ e de $\mathrm{K}$, por meio da injeção, na linha principal do sistema de irrigação, antes do sistema de filtragem. Utilizou-se como equipamento injetor uma bomba centrífuga de $0,5 \mathrm{cv}$, com rotor de Noryl $^{\circledR}$, instalada de maneira afogada. Esta promovia a sucção da solução composta de água e fertilizantes, a partir de um reservatório com capacidade de $150 \mathrm{~L}$. O tempo de fertirrigação foi de 30 minutos. O número de ramos plagiotrópicos foi avaliado através da contagem direta por planta. Estas avaliações foram realizadas em outubro de 2007 (697 dias após o plantio).

A colheita ocorreu em abril de 2008, de modo que a produtividade foi medida nas quatro unidades experimentais para cada tratamento. Para tal, derriçou-se o café colhido sobre um pano e, posteriormente, procedeuse a pesagem para a avaliação da massa de café em coco colhida em cada planta. Logo após, tomou-se amostras e realizou a correlação em massa de café em coco para café limpo. Em seguida, obteve-se a média de produção de cada unidade experimental, as quais foram convertidas em quilos por hectare, utilizando-se um fator relacionado com o estande de plantas que, neste caso, foi de 5000 plantas por hectare. Estes dados foram convertidos em número de sacas beneficiadas $(60 \mathrm{~kg})$ por hectare.

Tendo-se os dados, realizou-se a análise de variância. Na ocorrência de diferenças significativas, aplicou-se o teste de Scott-Knott, para as variáveis qualitativas (cultivos), e a análise de regressão, para as variáveis quantitativas (doses de NPK). Nestas análises foi utilizado o software estatístico Sisvar.

\section{Resultados e discussão}

\section{Número de ramos plagiotrópicos totais}

A observação dos dados da Tabela 1 permite dizer que os cultivos e doses de fertilizantes exerceram efeito significativo sobre o número de ramos plagiotrópicos totais da cultivar Obatã.

Os cultivos irrigados e fertirrigados trouxeram incrementos significativos ao número de ramos plagiotrópicos da cultivar de cafeeiro Obatã. Este comportamento pode ser explicado devido ao efeito sinérgico de uma adequada disponibilidade de água e de uma melhor distribuição dos nutrientes contidos nos fertilizantes, fato este proporcionado, principalmente, pela fertirrigação, conforme a interpretação dos dados contidos na Tabela 2 . 
Tabela 1 - Resumo da análise de variância, para a variável número total de ramos plagiotrópicos das cultivares de cafeeiro Obatã e IAPAR - 59

\begin{tabular}{lccc}
\hline \multirow{2}{*}{ Fontes de Variação } & GL & \multicolumn{2}{c}{ Cultivares } \\
\cline { 3 - 4 } & & Obatã & IAPAR - 59 \\
\cline { 3 - 4 } & 3 & $11,692^{*}$ & $6,751^{*}$ \\
\hline Doses de Fertilizantes & 2 & $3,300^{*}$ & $1,477^{\mathrm{Ns}}$ \\
Cultivos & 6 & $1,684^{*}$ & $2,894^{*}$ \\
Doses de Fertilizantes X Cultivos & & 45,40 & 48,90 \\
\hline Média Geral & 8,39 & 7,73 \\
\hline Coeficiente da Parcela (\%) & & 10,00 & 7,01 \\
\hline Coeficiente da Subparcela (\%) & & & \\
\hline
\end{tabular}

* significativo ao nível de $5 \%$ de probabilidade. ${ }^{\text {Ns }}$ não significativo ao nível de $5 \%$ de probabilidade

Tabela 2 - Valores médios obtidos para a característica número total de ramos plagiotrópicos totais (NTRP) para as cultivares de cafeeiro Obatã e IAPAR - 59

\begin{tabular}{ccc}
\hline \multirow{2}{*}{ Cultivos } & \multicolumn{2}{c}{ Cultivares } \\
\cline { 2 - 3 } & NTRP & NTRP \\
\cline { 2 - 3 } & Obatã & IAPAR-59 \\
\hline Não irrigado & $43,5 \mathrm{~b}$ & 48,2 \\
Irrigado & $45,6 \mathrm{a}$ & 49,0 \\
Fertirrigado & $45,9 \mathrm{a}$ & 49,5 \\
Média & 45,6 & 49,3 \\
\hline
\end{tabular}

Médias seguidas de letras distintas, na coluna, diferem entre si pelo teste de Scott-Knott ao nível de 5\% de probabilidade

A observação dos dados dispostos na Tabela 2 permite afirmar que o número de ramos plagiotrópicos da cultivar Obatã foi superior em cultivos irrigados e fertirrigados. Tais resultados corroboram com os obtidos por Santana et al. (2004), que concluíram que a cultivar Obatã destacou-se na avaliação desta variável.

A irrigação promoveu o aumento do número de ramos plagiotrópicos das plantas da cultivar Obatã. Tal comportamento corrobora os resultados obtidos por Carvalho et al. (2006) que, em um experimento realizado também com uma cultivar de Coffea arabica, sendo, neste caso, a cultivar Rubi MG - 1192, concluíram que as maiores médias desta componente de crescimento da cultura foram obtidas na condução de cafeeiros irrigados.

O estudo do desdobramento das doses de NPK, dentro de cada cultivo, mostrou que o número de ramos plagiotrópicos do cafeeiro Obatã, nas lavouras irrigadas e fertirrigadas, sofreu influência significativa da interação destes fatores.
O melhor ajuste das médias observadas, no cultivo irrigado, foi expresso através da regressão linear. Em relação às lavouras fertirrigadas do cafeeiro Obatã, a relação entre as diferentes doses de NPK aplicadas e seu número total de ramos plagiotrópicos pode ser explicada através da regressão quadrática. As estimativas dos coeficientes destes modelos de regressão polinomial estão dispostas na Tabela 3 e suas equações descritas na Figura 3.

Tabela 3 - Estimativa e significância dos parâmetros da regressão linear para o cultivo irrigado e da regressão quadrática para os cultivos fertirrigados do cafeeiro Obatã

\begin{tabular}{cccc}
\hline Cultivos & Parametros & Estimativa & Prob $>|\mathrm{t}|$ \\
\hline \multirow{2}{*}{ Irrigado } & $\beta_{0}$ & 42,15 & $0,0000^{*}$ \\
& $\beta_{1}$ & 0,0302 & $0,0141^{*}$ \\
\hline \multirow{3}{*}{ Fertirrigado } & $\beta_{0}$ & 49,775 & $0,0000^{*}$ \\
& $\beta_{1}$ & $-0,1517$ & $0,0293^{*}$ \\
& $\beta_{2}$ & 0,0008 & $0,0043^{*}$ \\
\hline
\end{tabular}

* significativo em um nível de $5 \%$ de probabilidade

Nos cultivos irrigados (FIG. 3A),o aumento da porcentagem de dose de NPK aplicada foi diretamente proporcional ao aumento do número de ramos plagiotrópicos, sendo mais adequada do ponto de vista de rendimento físico ou absoluto, neste caso, a dose de $200 \%$ que correspondeu à aplicação de $300 \mathrm{~kg} \mathrm{ha}^{-1}$ de $\mathrm{N}, 60 \mathrm{~kg} \mathrm{ha}^{-1}$ de $\mathrm{P}_{2} \mathrm{O}_{5}$ e $300 \mathrm{~kg} \mathrm{ha}^{-1}$ de $\mathrm{K}_{2} \mathrm{O}$. Estes resultados podem ser explicados devido ao fato de que a irrigação tende a potencializar o efeito das doses de fertilizantes aplicadas, mesmo quando são fornecidas tradicionalmente. 

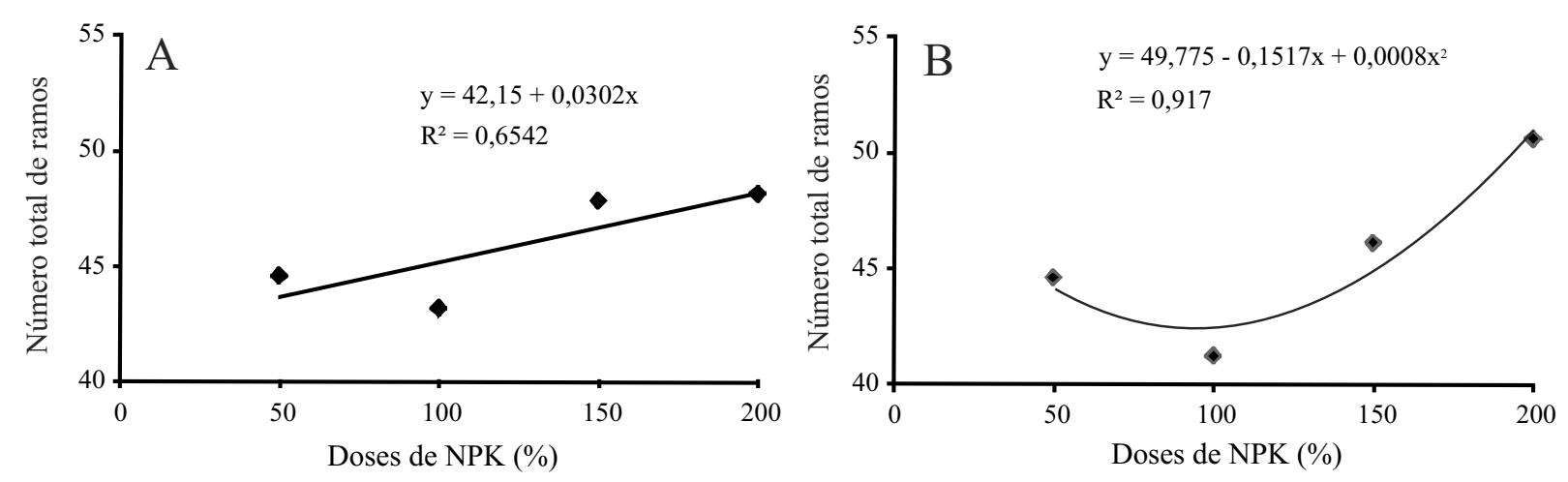

Figura 3 - Número total de ramos plagiotrópicos do cafeeiro nos cultivos irrigados (A) e fertirrigados (B) da cultivar Obatã em função das distintas porcentagens de doses de NPK aplicadas

De acordo com a observação da Figura 3B, podese afirmar que o número de ramos plagiotrópicos das plantas da cultivar Obatã, nos cultivos fertirrigados, diminuiu quando se aplicou $100 \%$ da dose de NPK sugerida pela literatura (MATIELLO et al., 2005) para, em seguida, aumentar com aplicação da dose de $150 \%$ e atingir um máximo com a aplicação da dose de percentual de $200 \%$.

As plantas da cultivar de cafeeiro IAPAR - 59 também sofreram o efeito da interação dos tratamentos, sendo que, somente nos cultivos irrigados, foram encontradas diferenças significativas relacionadas com as doses de fertilizantes. O modelo de regressão polinomial quadrático foi o que melhor representou as médias observadas, de modo que a estimativa de seus parâmetros está disposta na Tabela 4 e sua equação expressa na Figura 4.

A observação da Figura 4 permite afirmar que o número de ramos plagiotrópicos da cultivar IAPAR - 59 aumentou até a dose $150 \%$, na qual se obteve um máximo de 53 ramos com o fornecimento de $225 \mathrm{~kg} \mathrm{ha}^{-1}$ de $\mathrm{N}, 45 \mathrm{~kg} \mathrm{ha}^{-1}$ de $\mathrm{P}_{2} \mathrm{O}_{5}$ e $225 \mathrm{~kg} \mathrm{ha}^{-1}$ de $\mathrm{K}_{2} \mathrm{O}$ e diminuiu com a aplicação da dose de $200 \%$ da dose recomendada.

Tabela 4 - Estimativa e significância dos parâmetros da regressão quadrática para o número de ramos plagiotrópicos totais no cultivo irrigado da cultivar de cafeeiro IAPAR - 59

\begin{tabular}{cccc}
\hline Cultivo & Parametros & Estimativa & Prob $>|t|$ \\
\hline \multirow{3}{*}{ Irrigado } & $\beta_{0}$ & 32,3750 & $0,0000^{*}$ \\
& $\beta_{1}$ & 0,2933 & $0,0000^{*}$ \\
& $\beta_{2}$ & $-0,0011$ & $0,0000^{*}$ \\
\hline
\end{tabular}

* significativo em um nível de $5 \%$ de probabilidade

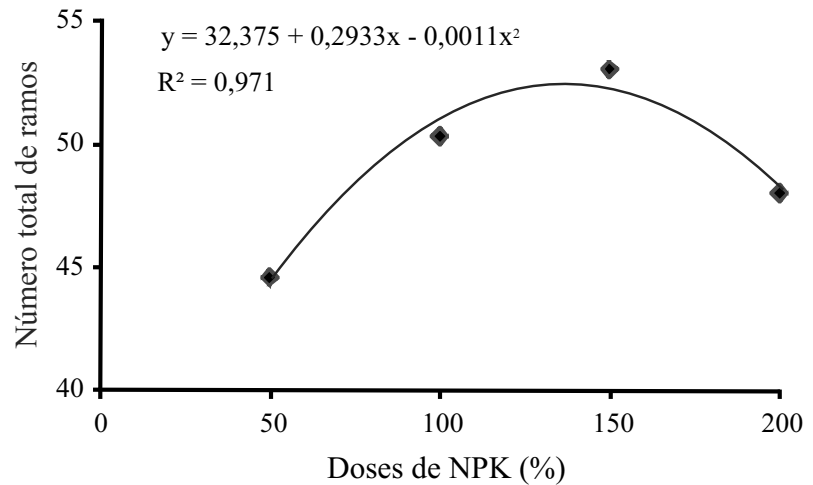

Figura 4 - Número total de ramos plagiotrópicos do cafeeiro nos cultivos irrigados da cultivar IAPAR - 59 em função das distintas porcentagens de doses de NPK aplicadas

Fazendo-se um estudo comparativo entre as doses de NPK de porcentagens $150 \%$ e $200 \%$, pode-se afirmar que se necessitou de uma menor quantidade de nutrientes para que as plantas das lavouras irrigadas da cultivar IAPAR - 59 pudessem produzir uma maior quantidade de ramos plagiotrópicos. Tal comportamento pode ter sido demonstrativo de que a irrigação favorece a movimentação dos nutrientes ao longo do perfil do solo, o que afeta positivamente a absorção de NPK pelas plantas de café.

\section{Produtividade}

De acordo com a observação dos dados da Tabela 5, os tratamentos doses de fertilizantes, cultivos, bem como a interação entre eles influíram significativamente nas médias de produtividade das cultivares Obatã e IAPAR - 59.

O estudo do desdobramento da análise de variância no qual se fixou os cultivos e se variou as doses 
Tabela 5 - Resumo da análise de variância, para a variável produtividade das cultivares de cafeeiro Obatã e IAPAR - 59

\begin{tabular}{lccc}
\hline \multirow{2}{*}{ Fontes de Variação } & GL & Cultivares \\
\cline { 3 - 4 } & & $\mathrm{F}$ & IAPAR - 59 \\
\cline { 3 - 4 } & 3 & $61,298^{*}$ & $100,281^{*}$ \\
Doses de Fertilizantes & 2 & $6028,738^{*}$ & $2836,817^{*}$ \\
Cultivos & 6 & $3,941^{*}$ & $2,548^{*}$ \\
\hline Moses de Fertilizantes X Cultivos & & 30,50 & 28,90 \\
\hline Coeficiente da Parcela (\%) & 2,24 & 2,30 \\
Coeficiente da Subparcela (\%) & 1,76 & 2,57 \\
\hline
\end{tabular}

* significativo ao nível de $5 \%$ de probabilidade

aplicadas de NPK apontou diferenças significativas nos cultivos (não irrigados, irrigados, fertirrigados) independente da cultivar analisada.

Desta forma, procedeu-se a análise de regressão. Em todos os casos, o melhor ajuste das médias observadas foi obtido com o modelo quadrático, sendo que seus coeficientes, para cada lavoura estudada da cultivar de cafeeiro Obatã, estão dispostos na Tabela 6 e suas equações para os cultivos não irrigados, irrigados e fertirrigados podem ser observadas na Figura 5.

Tabela 6 - Estimativa e significância dos parâmetros da regressão quadrática para a produtividade das lavouras da cultivar de cafeeiro Obatã

\begin{tabular}{cccc}
\hline Cultivos & Parametros & Estimativa & Prob $>|\mathrm{t}|$ \\
\hline Não irrigado & $\beta_{0}$ & 14,9250 & $0,0000^{*}$ \\
& $\beta_{1}$ & 0,0717 & $0,0000^{*}$ \\
& $\beta_{2}$ & $-0,0003$ & $0,0001^{*}$ \\
\hline Irrigado & $\beta_{0}$ & 26,5500 & $0,0000^{*}$ \\
& $\beta_{1}$ & 0,1210 & $0,0000^{*}$ \\
& $\beta_{2}$ & $-0,0004$ & $0,0000^{*}$ \\
\hline Fertirrigado & $\beta_{0}$ & 33,181 & $0,0000^{*}$ \\
& $\beta_{1}$ & 0,093 & $0,0000^{*}$ \\
& $\beta_{2}$ & $-0,0003$ & $0,0000^{*}$ \\
\hline
\end{tabular}

* significativo ao nível de $5 \%$ de probabilidade
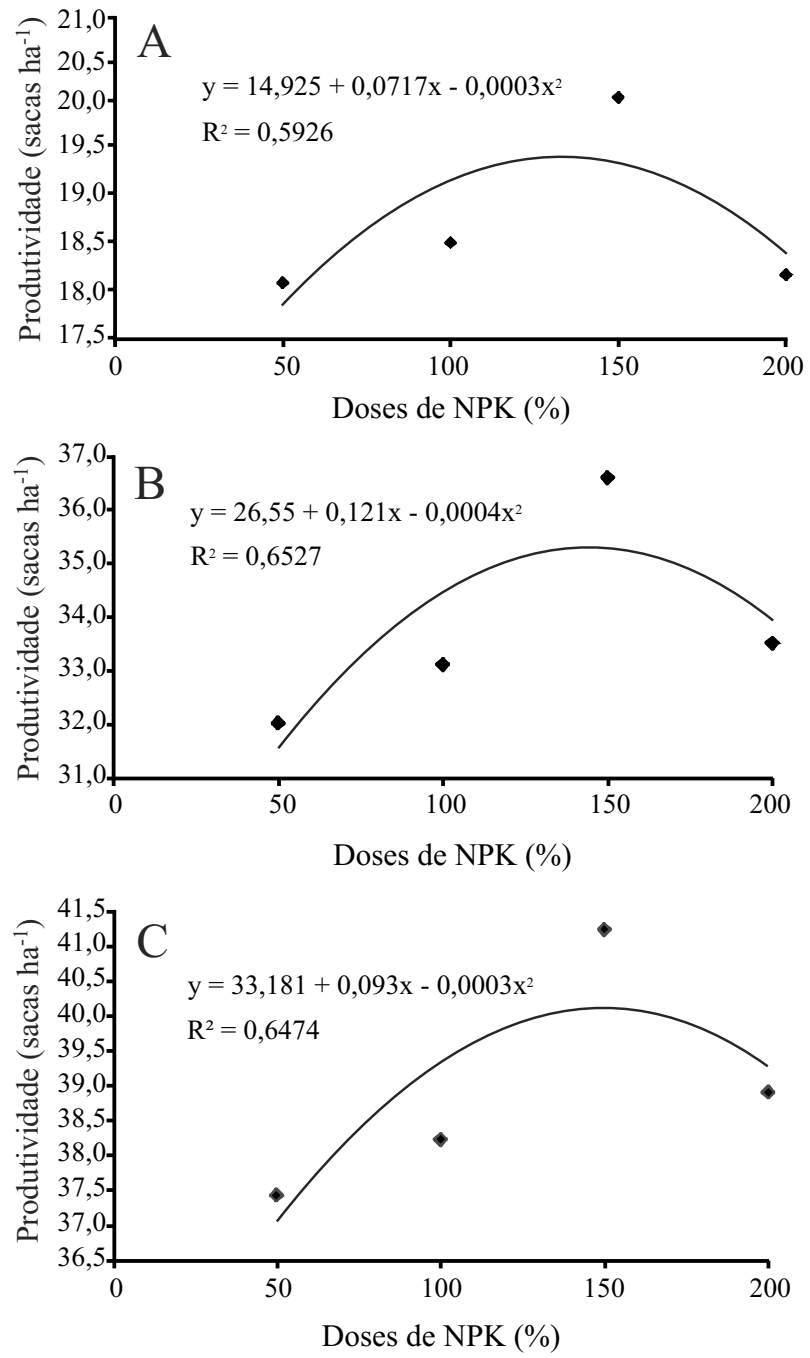

Figura 5 - Produtividade do cafeeiro nos cultivos não irrigados (A), irrigados (B) e fertirrigados (C) da cultivar Obatã, em função das distintas porcentagens de doses de NPK aplicadas 
A produtividade das plantas da cultivar de cafeeiro Obatã aumentou na medida em que se variaram às doses de $50 \%$ até a porcentagem de $150 \%$, em relação à sugerida pela literatura, na qual se obteve os melhores resultados e que correspondeu ao fornecimento da dose de $225 \mathrm{~kg} \mathrm{ha}^{-1}$ de N, $45 \mathrm{~kg} \mathrm{ha}^{-1} \mathrm{de}_{2} \mathrm{O}_{5}$ e $225 \mathrm{~kg} \mathrm{ha}^{-1}$ de $\mathrm{K}_{2} \mathrm{O}$. Este comportamento também se repetiu na produtividade da cultivar IAPAR - 59.

A máxima produtividade estimada para as plantas da cultivar de cafeeiro Obatã, no cultivo não irrigado, foi obtida com a dose estimada de $119,5 \%$, que correspondeu ao fornecimento estimado de 179, $25 \mathrm{~kg} \mathrm{ha}^{-1}$ de N, 35,85 $\mathrm{kg} \mathrm{ha}^{-1}$ de $\mathrm{P}_{2} \mathrm{O}_{5}$ e $179,25 \mathrm{~kg} \mathrm{ha}^{-1}$ de $\mathrm{K}_{2} \mathrm{O}$. Nos cultivos irrigados, a máxima produtividade estimada foi alcançada na dose estimada de $151,25 \%$, o que equivale a aplicação estimada de $226,875 \mathrm{~kg} \mathrm{ha}^{-1}$ de N, 45,375 $\mathrm{kg} \mathrm{ha}^{-1}$ de $\mathrm{P}_{2} \mathrm{O}_{5}$ e $226,875 \mathrm{~kg} \mathrm{ha}^{-1}$ de $\mathrm{K}_{2} \mathrm{O}$. Nos plantios fertirrigados, a produtividade máxima estimada foi obtida com a dose estimada de percentual $154,17 \%$, que compreendeu a aplicação de $231,25 \mathrm{~kg} \mathrm{ha}^{-1}$ de N,46,251 kg ha ${ }^{-1}$ de $\mathrm{P}_{2} \mathrm{O}_{5} \mathrm{e}$ $231,25 \mathrm{~kg} \mathrm{ha}_{-1}$ de $\mathrm{K}_{2} \mathrm{O}$.

Para a cultivar IAPAR - 59, a máxima produtividade estimada dentro dos cultivos não irrigados, irrigados e fertirrigados foi obtida nas doses de 137,83\%, 162\% e $151,75 \%$, respectivamente. Estes percentuais corresponderam a aplicação de $206,75 \mathrm{~kg} \mathrm{ha}^{-1}$ de $\mathrm{N}, 41,349 \mathrm{~kg} \mathrm{ha}^{-1}$ de $\mathrm{P}_{2} \mathrm{O}_{5}$, $206,75 \mathrm{~kg} \mathrm{ha}^{-1}$ de $\mathrm{K}_{2} \mathrm{O} ; 243 \mathrm{~kg} \mathrm{ha}^{-1}$ de N, 48,6 $\mathrm{kg} \mathrm{ha}^{-1} \mathrm{de}_{2} \mathrm{O}_{5}$, $243 \mathrm{~kg} \mathrm{ha}^{-1}$ de $\mathrm{K}_{2} \mathrm{O}$ e $227,625 \mathrm{~kg} \mathrm{ha}^{-1}$ de N, 45,525 $\mathrm{kg} \mathrm{ha}^{-1} \mathrm{de}$ $\mathrm{P}_{2} \mathrm{O}_{5}, 227,625 \mathrm{~kg} \mathrm{ha}^{-1}$ de $\mathrm{K}_{2} \mathrm{O}$, respectivamente.

Uma analise minuciosa das Figuras 5A, 5B e 5C permite dizer que a aplicação da dose de porcentagem de $200 \%$ trouxe incrementos a produtividade próximos aos trazidos pela dose de $100 \%$, a qual é recomendada pela literatura. Tal comportamento pode ser indicativo de que a aplicação da primeira dose citada seja inviável do ponto de vista econômico. Estas tendências também foram observadas na cultivar IAPAR - 59, sendo que a estimativa e significância dos componentes da regressão quadrática, para cada cultivo, estão dispostos na Tabela 7 e as equações para as lavouras não irrigadas, irrigadas e fertirrigadas são apresentadas na Figura 6.

Os resultados obtidos no presente ensaio são consoantes em parte com os encontrados por Prezotti e Rocha (2004) que tiveram por objetivo avaliar a produtividade de cafeeiros adubados com quatro doses de nitrogênio $(100 ; 300$; $\left.500 ; 700 \mathrm{~kg} \mathrm{ha}^{-1} \mathrm{de} \mathrm{N}\right)$, de fósforo $\left(0 ; 60 ; 120 ; 180 \mathrm{~kg} \mathrm{ha}^{-1} \mathrm{de}\right.$ $\mathrm{P}_{2} \mathrm{O}_{5}$ ) e de potássio $\left(100 ; 300 ; 500 ; 700 \mathrm{~kg} \mathrm{ha}^{-1}\right.$ de $\left.\mathrm{K}_{2} \mathrm{O}\right)$ em diversas densidades e espaçamentos de plantio utilizados: 20000 plantas por hectare $(1 \mathrm{~m} \mathrm{x} 0.5 \mathrm{~m}), 10000$ plantas por hectare $(2 \mathrm{~m} \mathrm{x} 0.5 \mathrm{~m}) ; 5000$ plantas por hectare $(2 \mathrm{~m} \mathrm{x} 1 \mathrm{~m})$; 3333 plantas por hectare $(3 \mathrm{~m} \times 1 \mathrm{~m})$. Ocorreram maiores respostas positivas para $\mathrm{N}$ e $\mathrm{P}$, nos diversos espaçamentos, e densidades e uma diminuição das respostas positivas a K,
Tabela 7 - Estimativa e significância dos parâmetros da regressão quadrática para a produtividade dos cultivos do cafeeiro IAPAR - 59

\begin{tabular}{lccc}
\hline Cultivos & Parametros & Estimativa & Prob $>|\mathrm{t}|$ \\
\hline \multirow{3}{*}{ Não irrigado } & $\beta_{0}$ & 12,70000 & $0,0000^{*}$ \\
& $\beta_{1}$ & 0,0827 & $0,0001^{*}$ \\
& $\beta_{2}$ & $-0,0003$ & $0,0003^{*}$ \\
\hline \multirow{2}{*}{ Irrigado } & $\beta_{0}$ & 24,1440 & $0,0000^{*}$ \\
& $\beta_{1}$ & 0,1296 & $0,0000^{*}$ \\
& $\beta_{2}$ & $-0,0004$ & $0,0000^{*}$ \\
\hline \multirow{3}{*}{ Fertirrigado } & $\beta_{0}$ & 29,0750 & $0,0000^{*}$ \\
& $\beta_{1}$ & 0,1214 & $0,0000^{*}$ \\
& $\beta_{2}$ & $-0,0004$ & $0,0000^{*}$ \\
\hline
\end{tabular}

* significativo ao nível de 5\% de probabilidade
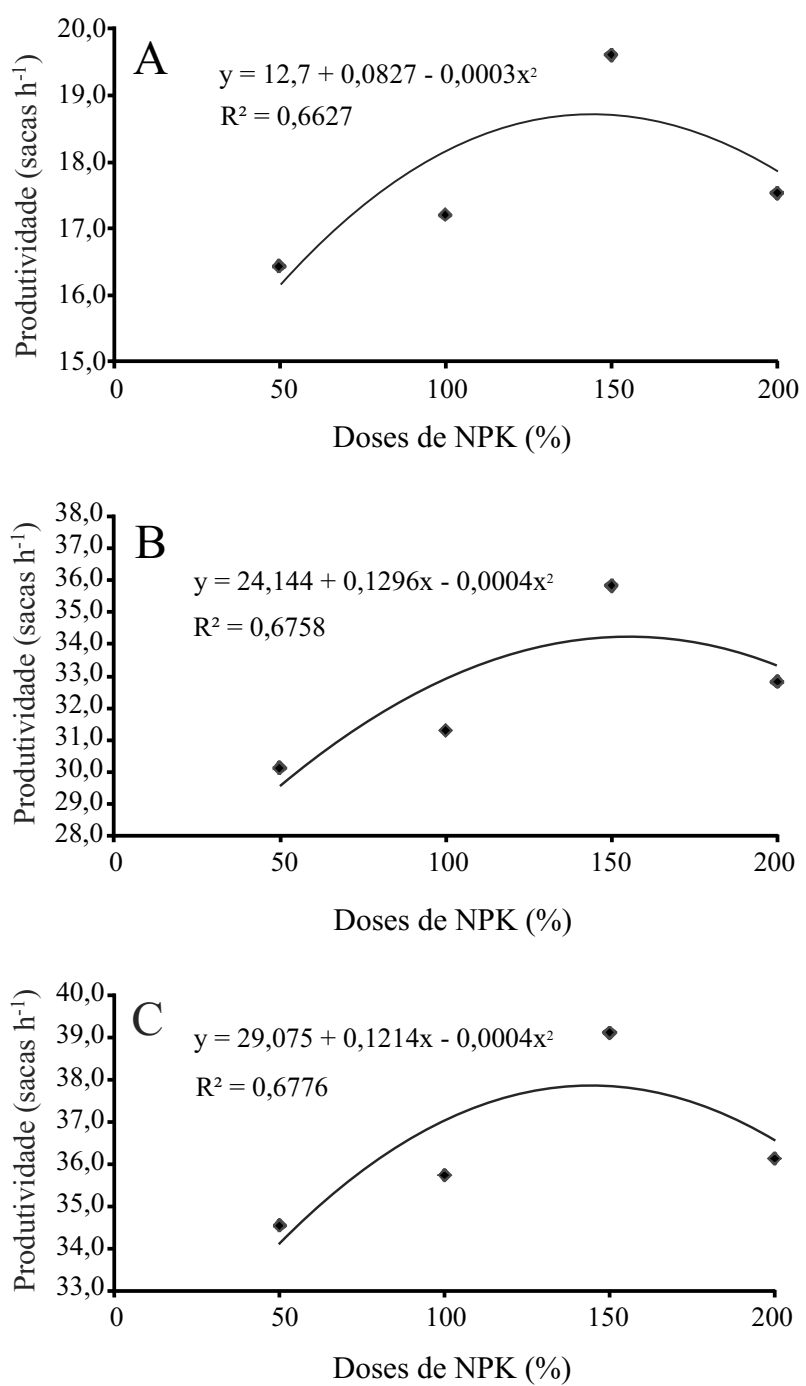

Figura 6 - Produtividade do cafeeiro nos cultivos não irrigados (A), irrigados (B) e fertirrigados (C) da cultivar IAPAR - 59, em função das distintas porcentagens de doses de NPK aplicadas 
em sistemas mais adensados, isto é, de menores densidades e maiores espaçamentos de plantio.

Uma analise minuciosa dos sinais dos parâmetros das equações da produtividade permite dizer que as funções de fato apresentam pontos de máximo, sendo que foi possível a determinação da porcentagem da dose de NPK que propiciou a máxima produtividade esperada em todos os cultivos das cultivares.

A Tabela 8 mostra os dados referentes à produtividade absoluta em sacas de $60 \mathrm{~kg}$, em função de todos os cultivos das cultivares Obatã e IAPAR - 59.

Tabela 8 - Valores médios obtidos para a característica produtividade (PROD), em função dos diferentes cultivos para as cultivares de cafeeiro IAPAR - 59 e Obatã

\begin{tabular}{ccc}
\hline \multirow{2}{*}{ Cultivos } & \multicolumn{2}{c}{ Cultivares } \\
\cline { 2 - 3 } & Obatã & IAPAR - 59 \\
\cline { 2 - 3 } & $\begin{array}{c}\text { Produtividade } \\
\left(\text { sacas ha }^{-1}\right)\end{array}$ & $\begin{array}{c}\text { Produtividade } \\
\left(\text { sacas ha }^{-1}\right)\end{array}$ \\
\hline Fertirrigado & $38,9 \mathrm{a}$ & $36,4 \mathrm{a}$ \\
Irrigado & $33,8 \mathrm{~b}$ & $32,5 \mathrm{~b}$ \\
Não irrigado & $18,8 \mathrm{c}$ & $17,7 \mathrm{c}$ \\
\hline
\end{tabular}

Médias seguidas de letras distintas, na coluna, diferem entre si pelo teste de Scott-Knott ao nível de 5\% de probabilidade

As maiores produtividades foram obtidas nos cultivos fertirrigados das duas cultivares, em comparação com os cultivos não irrigados, de modo que os incrementos conseguidos foram de $206 \%$ e $207 \%$ nas cultivares IAPAR - 59 e Obatã, respectivamente. Tais incrementos diferem dos obtidos por Antunes et al. (2000) que, em um experimento em Rio Preto - MG, verificaram que a produção obtida em cafeeiros, submetidos à fertirrigação, alcançou superioridade de $123 \%$, em comparação com os tratamentos não irrigados.

No plantio fertirrigado, foram aplicados como fontes de $\mathrm{K}$ e de $\mathrm{N}$ nitratos de potássio e de cálcio, respectivamente. As altas produtividades obtidas nestes cultivos podem estar relacionadas com a possibilidade do aumento da concentração de nitratos na água de irrigação devido à dissociação destes fertilizantes. Carrijo et al. (1999) afirmam que se os nitratos forem distribuídos em lâminas adequadas rapidamente serão absorvidos pelas plantas. É provável que o teor de nitratos na solução de solo tenha aumentado e isto pode ter favorecido a absorção de $\mathrm{K}^{+}, \mathrm{Ca}^{2+}$ e $\mathrm{Mg}^{2+}$, visto que, quando o $\mathrm{N}$ é absorvido na forma de nitrato $\left(\mathrm{NO}_{3}^{-}\right)$,ocorre a alcalinização da rizosfera da planta (CANTARELLA et al., 2007) e o aumento do
pH do solo (MALAVOLTA; MORAES, 2007), o qual pode contribuir para o aumento da concentração destes íons em sua solução.

No que diz respeito a $\mathrm{P}$, na cultura do café trata-se de um nutriente essencial para a produção dos frutos. É possível que tenha ocorrido resposta a $\mathrm{P}$ possivelmente por ele ter sido aplicado via água de irrigação diretamente na região radicular das plantas de cafeeiro. Como foi aplicado em forma prontamente disponível às plantas, ele pode ter tido pouco tempo de contato com o solo, o que limita sua adsorção, favorecendo sua disponibilidade na solução do solo (NOVAIS et al., 2007).

Neste trabalho, a produtividade dos cafeeiros fertirrigados diferiu significativamente da alcancada quando as cultivares foram apenas irrigadas e a produtividade obtida nestes dois cultivos foi estatisticamente superior a alcançada nos cultivos não irrigados, seja na cultivar IAPAR - 59 ou na cultivar Obatã. Este comportamento contraria parcialmente os resultados obtidos por Coelho et al. (2009) que, avaliando a produtividade de cafeeiros fertirrigados, irrigados e não irrigados, concluíram que não foram encontradas diferenças significativas entre a produtividade obtida no melhor tratamento fertirrigado em comparação com o tratamento irrigado. Contudo, o desempenho destes tratamentos foi superior ao alcançado em cafeeiros que não foram irrigados.

A irrigação trouxe incrementos significativos de $80 \%$ e $84 \%$ a produtividade das cultivares Obatã e IAPAR 59 , respectivamente, em relação aos cultivos não irrigados. Estes acréscimos aproximam-se dos obtidos por Fernandes et al. (2000) que, em um experimento em que submeteram cafeeiros a diversos regimes de irrigação, verificaram que o melhor tratamento de irrigação, que correspondeu ao fornecimento de água às plantas de cafeeiro durante os anos de 1995, 1996 e 1997, proporcionando deficiência hídrica nula trouxe ganhos a produtividade de até 101\%, em relação ao tratamento não irrigado.

Gomes et al. (2007), em um experimento, concluíram que a média de produtividade de cafeeiros irrigados ao final de cinco safras (safra 2000/2001, safra $2001 / 2002$, safra 2002/2003, safra 2003/2004, safra $2004 / 2005$ ) sofreu incrementos de $119 \%$, em comparação com a testemunha (cultivos de café não irrigados). Silva et al. (2008) avaliaram a produtividade de cafeeiros submetidos a três épocas de irrigação (A - 01/06/2003 a 30/09/2003, B - 01/09/2003 a 30/09/2003 e C - sem irrigação) e observaram que cafeeiros submetidos à irrigação, no período de junho a setembro de 2003, tiveram aumentos na produtividade de até $150 \%$, em comparação com cafeeiros não irrigados. 
É possível que as técnicas de fertirrigação tenham favorecido a absorção radicular de nitrogênio, fósforo e potássio pelos processos de fluxo em massa, principalmente, em relação ao primeiro e, em grande parte, pelo processo de difusão em relação aos dois últimos, respectivamente, fazendo - se o comparativo com o desempenho dos cultivos irrigados e não irrigados. Como foi utilizado o sistema de irrigação localizada por gotejamento, os elementos foram fornecidos em formas prontamente disponíveis na zona radicular das plantas de cafeeiro, de modo que a solução do solo nos cultivos fertirrigados torna-se mais rica em nutrientes, em comparação com a solução do solo nos cultivos irrigados. Na fertirrigação, as raízes das plantas terão acesso aos nutrientes carregados pela água de irrigação em conjunto com os contidos no solo, quando o mesmo é dissolvido, através da aplicação da solução fertilizante.

\section{Conclusões}

1. A prática da irrigação e da fertirrigação produziu incrementos na produtividade das duas cultivares. Entretanto, com relação ao número de ramos plagiotrópicos, os efeitos da irrigação e da fertirrigação se manifestaram somente nas plantas irrigadas e fertirrigadas de cafeeiro da cultivar Obatã;

2. No que diz respeito à produtividade máxima, as melhores doses de NPK para os cultivos não irrigados, irrigados e fertirrigados da cultivar Obatã são as de percentuais $119,5 \%, 151,25 \%$ e $154,17 \%$, respectivamente. Com relação a cultivar IAPAR - 59, a máxima produtividade estimada nos cultivos não irrigados, irrigados e fertirrigados correspondeu aos percentuais de $137,83 \%$, $162 \%$ e $151,75 \%$, respectivamente.

\section{Referências}

ANDRADE, B. M. de. Efeito da fertirrigação com nitrogênio e potássio sobre os teores de nutrientes em um solo cultivado com gravioleira. Revista Ciência Agronômica, v. 35, n. 02, p. 410-417, 2004.

ANTUNES, R. C. B. et al. Área de observação e pesquisa em cafeicultura irrigada na região das vertentes de Minas Gerais - resultados de 1998/2000. In: SIMPÓSIO DE PESQUISA DE CAFÉS DO BRASIL, 1., 2000, Poços de Caldas. Anais... Brasília: EMBRAPA CAFÉ/MINASPLAN, 2000. p. 823-826.

BONOMO, R. et al. Produtividade de cafeeiros arábica no cerrado goiano. Pesquisa Agropecuária Tropical, v. 38, n. 04, p. 233-240, 2008.

CANTARELLA, H. Nitrogênio. In: NOVAIS, R. F. et. al. Fertilidade do Solo. Viçosa - MG: Sociedade Brasileira de Ciência do Solo, 2007. 1017 p.
CARAMORI, P. H. et al. Zoneamento de riscos climáticos para a cultura de café (Coffea arabica L.) no estado do Paraná. Revista Brasileira de Agrometeorologia, v. 09, n. 03, p. 486-494, 2001.

CARRIJO, O. A. et al. Tendências e desafios da fertirrigação no Brasil. In: FOLEGATTI, M.V. (coord.) Fertirrigação: citrus, flores, hortaliças. Guaíba: Agropecuária, 1999. p. 155-169.

CARVALHO, C. H. M. de et al. Evolução do crescimento do cafeeiro (Coffea arabica L.) irrigado e não irrigado em duas densidades de plantio. Ciência e Agrotecnologia, v. 30, n. 02, p. 243-250, 2006.

COELHO, G. et al. Custo de produção da cafeicultura irrigada. Ciência e Agrotecnologia, p. 1534-1540, 2002. Edição especial.

COELHO, G. et al. Efeito de épocas de irrigação e de parcelamento de adubação sobre a produtividade do cafeeiro Catuaí. Ciência e Agrotecnologia, v. 33, n. 01, p. 67-73, 2009.

DUENHAS, L. H. et al. Fertirrigação com diferentes doses de NPK e seus efeitos sobre a produção e qualidade de frutos de laranja (Citrus sinensis O.) 'Valência'. Revista Brasileira de Fruticultura, v. 24, n. 01, p. 214-218, 2002.

EMPRESA BRASILEIRA DE AGROPECUÁRIA (EMBRAPA). Centro Nacional de Pesquisa de Solos. Sistema Brasileiro de Classificação de Solos. Rio de Janeiro: EMBRAPA/CNPSO, 1999. 412 p.

FERNANDES, A. L. T. et al. Deficiência hídrica e uso de granulados em lavoura cafeeira irrigada por gotejamento. Revista Brasileira de Engenharia Agrícola e Ambiental, v. 04, n. 03, p. 376-381, 2000.

GOMES, N. M.; LIMA, L. A.; CUSTÓDIO, A. A. de P. Crescimento vegetativo e produtividade do cafeeiro irrigado no sul do Estado de Minas Gerais. Revista Brasileira de Engenharia Agrícola e Ambiental, v. 11, n. 06, p. 564-570, 2007.

MALAVOLTA, E.; MORAES, M.F. Fundamentos do Nitrogênio e do Enxofre na Nutrição Mineral de Plantas Cultivadas. In: YAMADA, T.; ABDALLA, S. R. S.; VITTI, G. C. (Eds.). Nitrogênio e Enxofre na Agricultura Brasileira. Piracicaba: IPNI, 2007. p. 189-249.

MANTOVAnI, E. C.; VICENTE, M. R.; SOUZA, M. N. Caracterização técnica e perspectivas para a cafeicultura irrigada brasileira. In: ZAMBOLIM, L. (Org.). Efeitos da irrigação sobre a qualidade e produtividade do café. Viçosa, MG: [s.n.], 2004, p. 293-318. v. 1.

MATIELLO, J. B. et al. Cultura de café no Brasil: novo manual de recomendações. 5. ed. Rio de Janeiro: Ministério da Agricultura, da Pecuária e do Abastecimento - Fundação PROCAFÉ, 2005. 438 p.

NAZARENO, R. B. et al. Crescimento inicial do cafeeiro Rubi em resposta a doses de nitrogênio, fósforo e potássio e a regimes hídricos. Pesquisa Agropecuária Brasileira, v. 38, n. 08, p. 903-910, 2003.

NOVAIS, R. F. et al. Fertilidade do Solo. Viçosa: Sociedade Brasileira de Ciência do Solo, 2007. 1017 p. 
PREZOTTI, L. C.; ROCHA, A. C. da. Nutrição do cafeeiro arábica em função da densidade de plantas. Bragantia, v. 63, n. 02, p. 239-251, 2004.

SANTANA, M. S.; OLIVEIRA, C. A. de; QUADROS, M. Crescimento inicial de duas cultivares de cafeeiro adensado influenciado por níveis de irrigação localizada. Engenharia Agrícola, v. 24, n. 03, p. 644-653, 2004.

SANTINATO, R.; FERNANDES, A. L. T.; FERNANDES, D. R. Irrigação na cultura do café. 2 . ed. Belo Horizonte: $O$ Lutador, 2008. 474 p.

SILVA, A. L.; FARIA, M. A.; REIS, R. P. Viabilidade técnicoeconômica do uso do sistema de irrigação por gotejamento na cultura do cafeeiro. Revista Brasileira de Engenharia Agrícola e Ambiental, v. 07, n. 01, p. 37-44, 2003.

SILVA, A. M. da et al. Épocas de irrigação e parcelamento da adubação sobre a produtividade do cafeeiro, em quatro safras. Revista Brasileira de Engenharia Agrícola e Ambiental, v. 09, n. 03, p. 314-319, 2005.

SILVA, A. C. et al. Produtividade e potencial hídrico do cafeeiro Catuaí, em função da época de irrigação. Revista Brasileira de Engenharia Agrícola e Ambiental, v. 12, n. 01, p. 21-25, 2008.

VITTI, G. C.; BOARETO, A. E.; PENTEADO, S. R. Fertilizantes e fertirrigação. In: VITTI, G. C.; BOARETO, A. E. Fertilizantes Fluidos. Piracicaba: Potafos, 1994. cap. 06, p. 261-281. 